\title{
LISTA DE REVISTAS PERMUTADAS
}

A Comissão Editorial da revista Scientia Iuris, desde sua fundação, visou o relacionamento com instituições brasileiras e estrangeiras para o intercâmbio técnico, científico e cultural, de modo a expandir quantitativa e qualitativamente as atividades de ensino, pesquisa e extensão no âmbito das Ciências Jurídicas.

Assim, além da colaboração técnica e científica com entidades nacionais e internacionais, esta iniciativa permitiu o intercâmbio de publicações, as quais são incorporadas ao acervo de periódicos da Biblioteca Central da Universidade Estadual de Londrina (UEL) e disponibilizadas para toda comunidade universitária.

E para dar continuidade a cooperação mútua estabelecida com estas instituições, bem como para expandí-la, disponibilizamos a lista das revistas com as quais a Biblioteca Central mantém permuta.

\author{
AMICUS CURIAE: REVISTA DO CURSO DE DIREIT O DA UNESC \\ UNIVERSIDADE DO EXTREMO SUL CATARINENSE \\ BIBLIOTECA CENTRAL / INTERCÂMBIO \\ AV. UNIVERSITÁRIA, 1105 \\ CAIXA POSTAL, 3167 - BAIRRO UNIVERSITÁRIO \\ 86.806-000 - CRICIUMA - SC
}

ARGUMENTA: REVISTA DO PROGRAMA DE MESTRADO EM CIÊNCIA JURÍDICA DA FUNDINOPI

FACULDADE EST. DE DIREITO DO NORTE PIONEIRO

BIBLIOTECA CENTRAL / INTERCÂMBIO

AV. MANUEL RIBAS, 711 - CENTRO

CAIXA POSTAL, 103

86.400-000 - JACAREZINHO - PR

ARGUMENTUM: REVISTA DE DIREITO

UNIVERSIDADE DE MARÍLIA

BIBLIOTECA “ZILMA PARENTE DE BARROS”

AV. HYGINO MUZZI FILHO, 1.001

17.525-902 - MARÍLIA - SP

ARGUMENTUN JURE

CENTRO DE ENSINO SUPERIOR DE RONDONÓPOLIS

FAC. DO SUL DO MATO GROSSO

BIBLIOTECA CENTRAL / INTERCÂMBIO

AV. ARY COELHO, 829 - B. CIDADE SALMEM

78.705-050 - RONDONÓPOLIS - MT

BOLETIM DA FACULDADE DE DIREITO DA UNIVERSIDADE DE COIMBRA UNIVERSIDADE DE COIMBRA

BIBLIOTECA DA FACULDADE DE DIREITO 
PATIO DA UNIVERSIDADE, 3004-545

COIMBRA - PORTUGAL

\section{CADERNOS DIREITO GV}

REVISTA DIREITO GV

FUNDAÇÃO GETULIO VARGAS

ESCOLA DE DIREITO DE SÃO PAULO

BILBIOTECA CENTRAL / INTERCÂMBIO

RUA ROCHA, 233

01.330-000 - SÃO PAULO - SP

CIDADANIA E JUSTIÇA (ITUIUTABA)

UNIVERSIDADE DO ESTADO DE MINAS GERAIS

BIBLIOTECA CENTRAL / INTERCÂMBIO

RUA VEREADOR GERALDO MOISÉS DA SILVA, S/N

CAMPUS UNIVERSITÁRIO - CAIXA POSTAL, 431

38.302-192 - ITUIUTABA - MG

\section{CIÊNCIA E DIREITO: REVISTA JURÍDICA DA FIC-UNAES}

UNIÃO DA ASSOCIAÇÃO EDUCACIONAL SUL-MATOGROSSENSE

BIBLIOTECA CENTRAL / INTERCÂMBIO

RUA 26 DE AGOSTO, 63

79.002-080 - CAMPO GRANDE - MS

\section{CRÍTICA JURÍDICA}

FACULDADES DO BRASIL

BIBLIOTECA CENTRAL / INTERCÂMBIO

RUA KONRAD ADENAUER, 442

B. TARUMÃ

82.820-540 - CURITIBA - PR

DIREITO E JUSTIÇA

PONTÍFICIA UNIVERSIDADE CATÓLICA

BIBLIOTECA CENTRAL / INTERCÂMBIO

AV. IPIRANGA, 6.681

CAIXA POSTAL, 1.429

90.619-900 - PORTO ALEGRE - RS

DIREITO E PAZ

CENTRO UNIVERSITÁRIO SALESIANO DE SÃO PAULO

BIBLIOTECA CENTRAL / INTERCÂMBIO

RUA DOM BOSCO, 284

12.600-970 - LORENA - SP

\section{DIREITO E DEMOCRACIA}

OPINIO JURE

UNIVERSIDADE LUTERANA DO BRASIL

BIBLIOTECA CENTRAL / INTERCÂMBIO

AV. FARROUPILHA, 8.001

92.425-900 - CANOAS - RS 


\section{DIREITO \& JUSTIÇA}

UNIVERSIDADE REG. INT. ALTO URUGUAI DAS MISSÕES

BIBLIOTECA CENTRAL / INTERCÂMBIO

RUA UNIVERSIDADE DAS MISSÕES, 464

CAIXA POSTAL 203

98.802-470 - SANTO ANGELO - RS

\section{DIREITO E SOCIEDADE}

MINISTÉRIO PÚBLICO DO ESTADO DO PARANÁ

DIV. DE DOCUMENTAÇÃO E BIBLIOTECA

AV. MARECHAL FLORIANO PEIXOTO, 1.251

BAIRRO REBOUÇAS

80.230-110 - CURITIBA - PR

\section{DIREITO EM AÇÃO}

UNIVERSIDADE CATÓLICA DE BRASÍLIA

SISTEMA DE BIBLIOTECAS - BIBLIOTECA CENTRAL

QS. 07 - LOTE 01 - EPCT

ÁGUAS CLARAS - SETOR SUL

71.966-700 - TAQUATINGA - DF

\section{DISPUTATIONES: O DIREITO EM REVISTA}

FACULDADE DO NORTE NOVO DE APUCARANA

BIBLIOTECA CENTRAL / INTERCÂMBIO

AV. ZILDA SEIXAS AMARAL, 4.350

PQ. INDUSTRIAL

86.806-380 - APUCARANA - PR

\section{EM TEMPO (MARÍLIA)}

FUNDAÇÃO DE ENSINO "EURÍPIDES SOARES DA ROCHA" BIBLIOTECA CENTRAL AV. HYGINO MUZZI FILHO, 529

CAMPUS UNIVERSITÁRIO

17.525-901 - MARÍLIA - SP

EOS - REVISTA JURIDICA DA FACULDADE DE DIREITO

FACULDADE DOM BOSCO

BIBLIOTECA CENTRAL / INTERCÂMBIO

AV. PRES. WENCESLAU BRAZ, 1.172

CAMPUS II - MARUMBY

81.010-000 - CURITIBA - PR

\section{ESPAÇO JURÍDICO}

UNIVERSIDADE DO OESTE DE SANTA CATARINA

BIBLIOTECA CENTRAL / INTERCÂMBIO

RUA OIAPOC, 211 - BAIRRO AGOSTINI

CAMPUS SÃO MIGUEL DO OESTE

89.900-000 - SÃO MIGUEL DO OESTE - SC 


\section{ESTUDIOS DE DERECHO}

UNIVERSIDADE DE ANTIOQUIA

BIBLIOTECA CENTRAL / CANJE

CALE 67, N $\mathrm{N}^{\mathrm{a}}$ 53-108, BLOQUE 14, OFICINA 202

APARTADO AEREO 1226

MEDELLIN - COLOMBIA

ESTUDOS JURÍDICOS (SÃO LEOPOLDO)

UNIVERSIDADE DO VALE DO RIO DOS SINOS

BIBLIOTECA CENTRAL / INTERCÂMBIO

AV. UNISINOS, 950

93.022-000 - SÃO LEOPOLDO - RS

\section{FMU DIREITO:}

FACULDADES METROPOLITANA UNIDAS

BIBLIOTECA CENTRAL / INTERCÂMBIO

RUA TAGUA, 150 - LIBERDADE

01.508-010 - SÃO PAULO - SP

\section{INFOJUR}

TRIBUNAL REGIONAL FEDERAL DA $2^{\circ}$ REGIÃO

BIBLIOTECA

A/C- CÉSAR GONDIN - SUPERVISOR DE PERIÓDICOS

RUA DO ACRE, $80-8^{\mathrm{a}}$ ANDAR - CENTRO

20.081-000 - RIO DE JANEIRO - RJ

\section{INTERTEMAS: REVISTA DA TOLEDO}

ASSOCIAÇÃO EDUCACIONAL TOLEDO

BIBLIOTECA "VISCONDE DE SÃO LEOPOLDO"

PRAÇA RAUL FURQUIM, 09

VILA FURQUIM

19.030-430 - PRESIDENTE PRUDENTE - SP

JURIS POIÉSIS - REVISTA DO CURSO DE DIREITO DA UNIVERSIDADE

ESTÁCIO DE SÁ

UNIVERSIDADE ESTÁCIO DE SÁ

BIBLIOTECA CENTRAL / INTERCÂMBIO

RUA DO BISPO, 83

RIO COMPRIDO

20.261-060 - RIO DE JANEIRO - RJ

\section{JUS ET FIDES}

UNIVERSIDADE CATÓLICA DE PERNAMBUCO

BIBLIOTECA CENTRAL / INTERCÂMBIO

RUA DO PRINCIPE, 526

BAIRRO BOA VISTA

50.050-900 - RECIFE - PE 


\section{JUSTIÇA DO DIREITO}

UNIVERSIDADE DE PASSO FUNDO

BIBLIOTECA CENTRAL / INTERCÂMBIO

CAMPUS UNIVERSITÁRIO

CAIXA POSTAL, 611

99.001-970 - PASSO FUNDO - RS

\section{LUMIAR - REVISTA DE CIÊNCIAS JURÍDICAS}

UNIVERSIDADE ESTADUAL DE PONTA GROSSA

BIBLIOTECA CENTRAL / INTERCÂMBIO

PRAÇA SANTOS ANDRADE, 1 - CENTRO

84.010-790 - PONTA GROSSA - PR

\section{MATIZ}

INSTITUTO MATONENSE MUN. DE ENSINO SUPERIOR

BIBLIOTECA CENTRAL / INTERCÂMBIO

AV. TIRADENTES, 629 - CENTRO

15.990-185 - MATÃO - SP

\section{MERITUM}

FACULDADE DE CIÊNCIAS HUMANAS DA

UNIVERSIDADE FUMEC

BIBLIOTECA CENTRAL / INTERCÂMBIO

RUA COBRE, 200 - BAIRRO CRUZEIRO

30.310-190 - BELO HORIZONTE - MG

\section{NOTÍCIA DO DIREITO BRASILEIRO}

UNIVERSIDADE DE BRASÍLIA

BIBLIOTECA CENTRAL / INTERCÂMBIO

CAMPUS UNIVERSITÁRIO "DARCY RIBEIRO"

GLEBA "A" - C. P. 15.299

70.910-900 - BRASÍLIA - DF

\section{NOVOS ESTUDOS JURÍDICOS}

UNIVERSIDADE DO VALE DO ITAJAI

BIBLIOTECA CENTRAL / INTERCÂMBIO

RUA URUGUAI, 458

CAIXA POSTAL, 360

88.302-202 - ITAJAI - SC

PENSAR: REVISTA DO CURSO DE DIREITO DA UNIVERSIDADE DE FORTALEZA UNIVERSIDADE DE FORTALEZA

FUNDAÇÃO EDUCACIONAL "EDSON QUEIROZ"

BIBLIOTECA CENTRAL / INTERCÂMBIO

AV. WASHINGTON SOARES, 1.321 - C. P. 1.258

60.811-905 - FORTALEZA - CE

\section{PRISMA JURÍDICO}

UNIVERSIDADE NOVE DE JULHO

SISTEMA BIBLIOTECAS”PROF. JOSÉ STORÓPOLI" 
RUA DIAMANTINA, 302 - VILA MARIA

02.117-010 - SÃO PAULO - SP

\section{REVISTA BONIJURIS}

INSTITUTO DE PESQUISAS JURÍDICAS BONIJURIS

A/C-BIBLIOTECA CENTRAL

RUA MARECHAL DEODORO, 344 - $3^{\mathrm{a}}$ ANDAR

CENTRO

80.010-909 - CURITIBA - PR

REVISTA DE CIÊNCIAS JURÍDICAS (MARINGÁ)

REVISTA DE DIREITO PRIVADO E PROCESUAL

UNIVERSIDADE ESTADUAL DE MARINGÁ

BIBLIOTECA CENTRAL / INTERCÂMBIO

AV. COLOMBO, 5.790

87.020-900 - MARINGÁ - PR

REVISTA DE CIÊNCIAS JURÍDICAS E SOCIAIS DA UNIPAR

UNIVERSIDADE PARANAENSE

BIBLIOTECA CENTRAL / INTERCÂMBIO

AV. PARIGOT DE SOUZA, 3.636 - JD. PRADA

85.903-170 - TOLEDO - PR

REVISTA DE CI ÊNCIAS JUR ÍDI CAS E SOCIAIS DA UNIPAR

UNIVERSIDADE PARANAENSE

BIBLIOTECA CENTRAL / INTERCÂMBIO

PRAÇA MASCARENHAS DE MORAES, 4.282

87.502-210 - UMUARAMA - PR

REVISTA DO CURSO DE DIREITO DA FSG

FACULDADE DA SERRA GAÚCHA

BIBLIOTECA CENTRAL / INTERCÂMBIO

RUA OS DEZOITO DO FORTE, 2.366

95.020-472 - CAXIAS DO SUL - RS

\section{REVISTA DAEMERJ}

ESCOLA DE MAGISTRATURA DO ESTADO RIO DE JANEIRO

BIBLIOTECA - AT. MARIA BEATRIZ

AV. ERASMO BRAGA, 115 - $4^{\text {a }}$ ANDAR - CENTRO

20.020-903 - RIO DE JANEIRO - RJ

\section{REVISTA DE DIREITO DAADVOCEF}

ASSOCIAÇÃO NACIONAL DOS ADVOGADOS DA

CAIXA ECONÔMICA FEDERAL

BIBLIOTECA

RUA SANTA CATARINA, 50 - SALAS 602/603

86.010-470 - LONDRINA - PR

REVISTA DE DIREITO DA CONCORRÊNCIA

MINISTÉRIO DA JUSTIÇA 
CONSELHO ADMINISTRATIVO DE DEFESA ECONOMICA

SCN QUADRA 2 PROJEÇÃO C

70.712-902 - BRASÍLIA - DF

\section{REVISTA DIREITO MACKENZIE}

INSTITUTO PRESBITERIANO MACKENZIE

BIBLIOTECA "GEORGE ALEXANDER" / INTERCÂMBIO

RUA DA CONSOLAÇÃO, 896 - PRÉDIO 02 - CONSOLAÇÃO

01.302-907 - SÃO PAULO - SP

\section{REVISTA DE DIREITO UPIS}

FACULDADES INTEGRADAS DA UNIÃO PIONEIRA

DE INTEGRAÇÃO SOCIAL

BIBLIOTECA CENTRAL / INTERCÂMBIO

SEP SUL - EQ 712/912 - CONJ. A

70.390-125 - BRASÍLIA - DF

\section{REVISTA DO CURSO DE DIREITO}

ASSOCIAÇÃO ENSINO UNIFICADO DO DISTRITO FEDERAL

BIBLIOTECA CENTRAL / INTERCÂMBIO

SEUP - EQS 704/904 - CONJ. A

70.390-045 - BRASÍLIA - DF

REVISTA DO CURS O DE DIREITO DA UNIVERSIDADE FEDERAL DE UBERLÂNDIA

UNIVERSIDADE FEDERAL DE UBERLÂNDIA

SETOR DE AQUISIÇÃO

AV. JOÃO NAVES DE AVILA, 2.160 - BL. 3C

CAMPUS SANTA MONICA

38.408-100 - UBERLÂNDIA - MG

\section{REVISTA DO DIREITO (CACHOEIRA DE ITAPEMIRIM)}

FACULDADE DE DIREITO DE CACHOEIRA DO ITAPEMIRIM

BIBLIOTECA CENTRAL / INTERCÂMBIO

RUA MARIO IMPERIAL, 56 - B. DOS FERROVIÁRIOS

CAIXA POSTAL, 14

29.308-400 - CACHOEIRA DO ITAPEMIRIM - ES

\section{REVISTA DO DIREITO (SANTA CRUZ DO SUL)}

UNIVERSIDADE DE SANTA CRUZ DO SUL

BIBLIOTECA CENTRAL / INTERCÂMBIO

A/C- VALÉRIA FAVA DAL OSTO

CAMPUS UNIVERSITÁRIO

96.815-900 - SANTA CRUZ DO SUL - RS

\section{REVISTA DA FACULDADE CHRISTUS}

FACULDADE CHRISTUS

BIBLIOTECA CENTRAL / INTERCÂMBIO

RUA ISRAEL BEZERRA, 630

B. DIONIZIO TORRES

60.135-460 - FORTALEZA - CE 


\section{REVISTA FACULDADE DE DIREITO MILTON CAMPOS \\ FACULDADE DE DIREITO MILTON CAMPOS \\ BIBLIOTECA CENTRAL / INTERCÂMBIO \\ RUA MILTON CAMPOS, 202 \\ BAIRRO VILA DA SERRA - CAMPUS I \\ 34.000-000 - NOVA LIMA - MG}

\section{REVISTA DA FACULDADE DE DIREITO UNIRITTER}

FACULDADES INT. DO INSTITUTO RITTER DOS REIS

BIBLIOTECA CENTRAL / INTERCÂMBIO

RUA SANTOS DUMONT, 888 - B. NITERÓI

CAIXA POSTAL, 358

92.120-110 - CANOAS - RS

\section{REVISTA DA FACULDADE DE DIREITO UNIRITTER}

FACULDADES INT. DO INSTITUTO RITTER DOS REIS

BIBLIOTECA CENTRAL / INTERCÂMBIO

RUA ORFANOTRÓFIO, 555

CAIXA POSTAL, 1.355

90.840-440 - PORTO ALEGRE - RS

REVISTA DA FACULDADE DE DIREITO DE SÃO BERNARDO CAMPO FACULDADE DE DIREITO DE SÃO BERNARDO DO CAMPO BIBLIOTECA CENTRAL / INTERCÂMBIO

RUA JAVA, 425 - JD. DO MAR

09.750-650 - SÃO BERNARDO DO CAMPO - SP

REVISTA DA FACULDADE DE DIREITO UFG

UNIVERSIDADE FEDERAL DE GOIÁS

BIBLIOTECA CENTRAL / INTERCÂMBIO

CAMPUS SAMAMBAIA

CAIXA POSTAL 411

74.001-970 - GOIÂNIA - GO

\section{REVISTA DA FACULDADE DE DIREIT O DA UNIVERSIDADE FEDERAL} DO RIO GRANDE DO SUL

UNIVERSIDADE FEDERAL DO RIO GRANDE DO SUL

FACULDADE DE DIREITO

BIBLIOTECA SETORIAL

AV. JOÃO PESSOA, $80-2^{\circ}$ PAVIMENTO

90.040-000 - PORTO ALEGRE - RS

REVISTAJURÍDICA(GUARAPUAVA)

FACULDADE NOVO ATENEU DE GUARAPUAVA

BIBLIOTECA CENTRAL / INTERCÂMBIO

RUA SALDANHA MARINHO, 1.706 - CENTRO

85.010-290 - GUARAPUAVA - PR 


\section{REVISTAJURÍDICA(FOZ DO IGUAÇU)}

FACULDADES UNIFICADAS DE FOZ DO IGUAÇU

BIBLIOTECA CENTRAL / INTERCÂMBIO

RUA XAVIER DA SILVA, 775

85.851-180 - FOZ DO IGUAÇU - PR

\section{REVISTAJURÍDICA (FREDERICO WESTPHALEN)}

UNIVERSIDADE REG. INT. ALTO URUGUAI DAS MISSÕES

BIBLIOTECA CENTRAL / INTERCÂMBIO

AV. ASSIS BRASIL, 709 - CAIXA POSTAL, 184

CAMPUS FREDERICO WESTPHALEN

98.400-000 - FREDERICO WESTPHALEN - RS

\section{REVISTAJURÍDICA}

ASSOCIAÇÃO EDUCATIVA EVANGÉLICA

BIBLIOTECA CENTRAL / INTERCÂMBIO

AV. UNIVERSITÁRIA, KM 3,5

CIDADE UNIVERSITÁRIA

75.070-290 - ANAPOLIS - GO

\section{REVISTAJURÍDICA DA FADISMA}

FACULDADE DE DIREITO DE SANTA MARIA

BIBLIOTECA CENTRAL / INTERCÂMBIO

RUA DUQUE DE CAXIAS, 2.139

BAIRRO MEDIANEIRA

97.060-210 - SANTA MARIA - RS

\section{REVISTAJURÍDICA DA FAMINAS}

FACULDADE DE MINAS

BIBLIOTECA CENTRAL

AV. CRISTIANO FERREIRA VARELLA, 655

BAIRRO UNIVERSITÁRIO - C. P. 351

36.880-000 - MURIAÉ - MG

REVISTAJURÍDICA DA UNIVERSIDADE DE CUIABÁ

ENSAIOS ACADÊMICOS DA FACULDADE DE DIREITO DA UNIVERSIDADE DE

CUIABÁ

UNIVERSIDADE DE CUIABÁ

BIBLIOTECA CENTRAL / INTERCÂMBIO

AV. BEIRA RIO, 3.100 - JD. EUROPA

78.015-480 - CUIABÁ - MT

REVISTAJURÍDICADAUNIFIL

CENTRO UNIVERSITÁRIO FILADELFIA

BIBLIOTECA PROF. ZAQUEU DE MELO

RUA ALAGOAS, 2.050

CENTRO

86.020-430 - LONDRINA - PR 


\section{REVISTA JURÍDICA DO CESUT}

CENTRO ENSINO SUPERIOR DE JATAÍ

BIBLIOTECA CENTRAL / INTERCÂMBIO

RUA SANTOS DUMONT, 1.200 - SETOR OESTE

CAIXA POSTAL, 182

75.804-045 - JATAÍ - GO

\section{REVISTA JURÍDI CA(CAMPO GRANDE)}

UNIVERSIDADE CATÓLICA DOM BOSCO

BIBLIOTECA CENTRAL / INTERCÂMBIO

AV. TAMANDARÉ, 6.000

CAIXA POSTAL, 100

79.117-010 - CAMPO GRANDE - MS

\section{REVISTAJURÍDICAUNIDERP}

UNIVERSIDADE PARA O DESENVOLVIMENTO DO

ESTADO E DA REGIÃO DO PANTANAL

BIBLIOTECA CENTRAL / INTERCÂMBIO

R. CEARÁ, 333 - MIGUEL COUTO - C. P. 2.153

79.003-010 - CAMPO GRANDE - MS

\section{REVISTAJURÍDICA UNIJUS}

UNIVERSIDADE DE UBERABA

BIBLIOTECA CENTRAL / INTERCÂMBIO

AV. NENÊ SABINO, 1.801

BAIRRO UNIVERSITÁRIO

38.055-500 - UBERABA - MG

REVISTA JURÍDICA DA UNIVERSIDADE DE FRANCA

UNIVERSIDADE DE FRANCA

BIBLIOTECA CENTRAL / INTERCÂMBIO

AV. DR. ARMANDO SALLES OLIVEIRA, 201

PARQUE UNIVERSITÁRIO

14.404-600 - FRANCA - SP

\section{REVISTA JURÍDICA UNOESC}

UNIVERSIDADE DO OESTE DE SANTA CATARINA

BIBLIOTECA CENTRAL / INTERCÂMBIO

RUA GETULIO VARGAS, 2.125 - B. FLOR DA SERRA

CAMPUS DE JOAÇABA

89.600-000 - JOAÇABA - SC

\section{REVISTA MESTRADO EM DIREITO}

CENTRO UNIVERSITÁRIO FIEO

BIBLIOTECA - SETOR DE PERIÓDICOS

AV. FRANZ VOEGELI, 300 - BL. MARROM - SUBSOLO

VILAYARA

06.020-190 - OSASCO - SP 


\section{REVISTA DA FACULDADE MINEIRA DE DIREITO}

PONTIFÍCIA UNIVERSIDADE CATÓLICA BIBLIOTECA PE. ALBERTO ANTONIAZZI AV. DOM JOSÉ GASPAR, 500 - PRÉDIO 26 CORAÇÃO EUCARÍSTICO 30.535-901 - BELO HORIZONTE - MG

\section{REVISTA DA FACULDADE DE DIREITO}

FACULDADES INTEGRADAS DE GUARULHOS BIBLIOTECA CENTRAL / INTERCÂMBIO RUA DR. SOLAN FERNANDES, 155

CAMPUS VILA ROSÁLIA 07.072-080 - GUARULHOS - SP

\section{REVISTA DA FACULDADE DE DIREITO} UNIVERSIDADE FEDERAL DO PARANÁ BIBLIOTECA CENTRAL / INTERCÂMBIO CAIXA POSTAL, 19.051

81.531-990 - CURITIBA - PR

\section{REVISTA DA FACULDADE DE DIREITO}

UNIVERSIDADE FEDERAL DE PELOTAS

BIBLIOTECA CENTRAL / INTERCÂMBIO

CAMPUS UNIVERSITÁRIO

CAIXA POSTAL, 354

96.010-900 - PELOTAS - RS

Revista da Faculdade de Direito Universidade Federal Minas Gerais UNIVERSIDADE FEDERAL DE MINAS GERAIS

FACULDADE DE DIREITO - BIBLIOTECA AV. JOÃO PINHEIRO, 100 - CENTRO

30.130-180 - BELO HORIZONTE - MG

\section{REVISTA DA FACULDADE DE DIREITO DE CAMPOS}

FACULDADE DE DIREITO DE CAMPOS BIBLIOTECA CENTRAL / INTERCÂMBIO RUA TENENTE CORONEL CARDOSO, 349 28.013-460 - CAMPOS DE GOYTACAZES - RJ

REVISTA JURÍDICA DA FACULDADE DE DIREITO DE SOROCABA FACULDADE DE DIREITO DE SOROCABA BIBLIOTECA CENTRAL / INTERCÂMBIO RUA DR ${ }^{\mathrm{a}}$ URSULINA LOPES TORRES, 123 18.030-103 - SOROCABA - SP

REVISTA DAFACULDADE DE DIREITO UFC UNIVERSIDADE FEDERAL DO CEARÁ BIBLIOTECA DE DIREITO RUA METON DE ALENCAR, S/N - CENTRO 60.035-160 - FORTALEZA - CE 


\section{REVISTA BRASILEIRA DE DIREITO COMPARADO}

INSTITUTO DE DIREITO COMPARADO LUSO BRASILEIRO

BIBLIOTECA CENTRAL / INTERCÂMBIO

AV. N. SR ${ }^{\mathrm{a}}$ DE COPACABANA, 1.183 - SALA 503

22.070-011 - RIO DE JANEIRO - RJ

\section{REVISTA DE ESTUDOS JURÍDICOS}

UNIVERSIDADE SALGADO DE OLIVEIRA

BIBLIOTECA CENTRAL / INTERCÂMBIO

RUA MARECHAL DEODORO, 217 - CENTRO

24.030-060 - NITERÓI - RJ

REVISTA DO CURSO DE DIREITO DA UNIVERSIDADE FEDERAL SANTA MARIA

UNIVERSIDADE FEDERAL DE SANTA MARIA

CENTRO DE CIÊNCIAS SOCIAIS E HUMANAS

BIBLIOTECA SETORIAL / CCSH

RUA FLORIANO PEIXOTO, 1.184 - SALA 101

97.015-372 - SANTA MARIA - RS

\section{REVISTA DO MESTRADO EM DIREITO}

UNIVERSIDADE FEDERAL DE ALAGOAS

BIBLIOTECA CENTRAL / INTERCÂMBIO

CAMPUS UNIVERSITÁRIO A C. SIMÕES

BR 104, KM 97 - TABULEIRO DO MARTINS

57.072-970 - MACEIÓ - AL

\section{REVISTA DO PROGRAMA DE PÓS-GRADUAÇÃO EM DIREITO DA}

UNIVERSIDADE DAAMAZÔNIA

UNIVERSIDADE DA AMAZÔNIA

BIBLIOTECA CENTRAL / INTERCÂMBIO

AV. ALCINDO CACELA, 287

66.060-902 - BELÉM - PA

\section{REVISTAJURÍDICA- UNISEP}

UNIÃO DE ENSINO DO SUDOESTE DO PARANÁ

BIBLIOTECA CENTRAL / INTERCÂMBIO

AV. PRESIDENTE KENEDY, 2.601

BAIRRO NOSSA SR ${ }^{\mathrm{a}}$ APARECIDA

85.660-000 - DOIS VIZINHOS - PR

REVISTAJURÍDICA(BLUMENAU)

UNIVERSIDADE REGIONAL DE BLUMENAU

BIBLIOTECA CENTRAL / INTERCÂMBIO

RUA ANTONIO DA VEIGA, 140

CAIXA POSTAL, 1.507

89.012-900 - BLUMENAU - SC

REVISTA JURÍDICA CESUMAR MESTRADO

CENTRO UNIVERSITÁRIO DE MARINGÁ 
BIBLIOTECA CENTRAL / INTERCÂMBIO

AV. GUEDNER, 1.610

JD. ACLIMAÇÃO

87.050-390 - MARINGÁ - PR

\section{REVISTA EPD}

ESCOLA PAULISTA DE DIREITO

BIBLIOTECA

AV. LIBERDADE, 808

BAIRRO DA LIBERDADE

01.502-001 - SÃO PAULO - SP

\section{REVISTAJURÍDICA IN VERBIS}

UNIVERSIDADE FEDERAL DO RIO GRANDE DO NORTE

BIBLIOTECA CENTRAL / INTERCÃMBIO

CAMPUS UNIVERSITÁRIO - C. P. 1.524

59.072-970 - NATAL - RN

\section{REVISTAJURÍDICAUNIGRAN}

CENTRO UNIVERSITÁRIO DA GRANDE DOURADOS

BIBLIOTECA CENTRAL / INTERCÂMBIO

RUA BALBINA DE MATOS,, 2.121 - BL. 03

CAMPUS UNIGRAN

79.824-900 - DOURADOS - MS

REVISTA TRABALHO E AMBIENTE

UNIVERSIDADE DE CAXIAS DO SUL

BIBLIOTECA CENTRAL / INTERCÂMBIO

RUA FRANCISCO GETULIO VARGAS, 1.130

95.070-560 - CAXIAS DO SUL - RS

SEQUENCIA: REVISTA DO CURSO DE PÓS-GRADUAÇÃO DA UFSC

UNIVERSIDADE FEDERAL DE SANTA CATARINA

BIBLIOTECA UNIVERSITÁRIA

SETOR DE INTERCÂMBIO

CAIXA POSTAL, 476

88.010-970 - FLORIANÓPOLIS - SC

\section{SIGNUM}

CENTRO DE ENSINO SUPERIOR DE VITÓRIA

BIBLIOTECA CENTRAL / INTERCÂMBIO

RUA WELLINGTON DE FREITAS, 265

JD. CAMBURI

29.090-240 - VITÓRIA - ES

\section{THEMIS: REVISTA DE DIREITO}

UNIVERSIDADE NOVA DE LISBOA

FACULDADE DE DIREITO - BIBLIOTECA

CAMPUS DE CAMPOLIDE

TRAVESSA ESTEVÃO PINTO

1099-032 - LISBOA - PORTUGAL 
UNIVERSITÁRIA: REVISTA DO CURSO DE MESTRADO EM DIREITO

CENTRO UNIVERSITÁRIO TOLEDO

BIBLIOTECA CENTRAL / INTERCÂMBIO

RUA ANTONIO AFONSO DE TOLEDO, 595

BAIRRO JARDIM SUMARÉ

16.015-270 - ARAÇATUBA - SP

UNIVERSITAS /JUS

CENTRO UNIVERSITÁRIO DE BRASÍLIA

BIBLIOTECA CENTRAL / INTERCÂMBIO

SEPN 707/907 - CAMPUS DO CEUB - ASA NORTE

70.790-075 - BRASÍLIA - DF

UNOPAR CIENTÍFICA: CIÊNCIAS JURÍDICAS E EMPRESARIAIS

UNIVERSIDADE DO NORTE DO PARANÁ

BIBLIOTECA CENTRAL / INTERCÂMBIO

AV. PARIS, 675 - JD. PIZA

86.041-100 - LONDRINA - PR

VERBA JURIS: ANUÁRIO DA PÓS-GRADUAÇÃO EM DIREITO

UNIVERSIDADE FEDERAL DA PARAÍBA

CENTRO CIÊNCIAS JURÍDICAS

AV. GAL. OSÓRIO, 415

ED. BANCO REAL, $5^{\mathrm{a}}$ ANDAR

58.010-780 - JOÃO PESSOA - PB

VEREDAS DO DIREITO

ESCOLA SUPERIO DOM HELDER CAMARA

BIBLIOTECA CENTRAL

R. ÁLVARESS MACIEL, 628

B. SANTA EFIGÊNIA

30.150-250 - BELO HORIZONTE - MG

\section{VERITATI}

UNIVERSIDADE CATÓLICA DE SALVADOR

BIBLIOTECA CENTRAL / INTERCÂMBIO

AV. CARDEAL DA SILVA, 205

CAMPUS DA FEDERAÇÃO

40.231-902 - SALVADOR - BA 